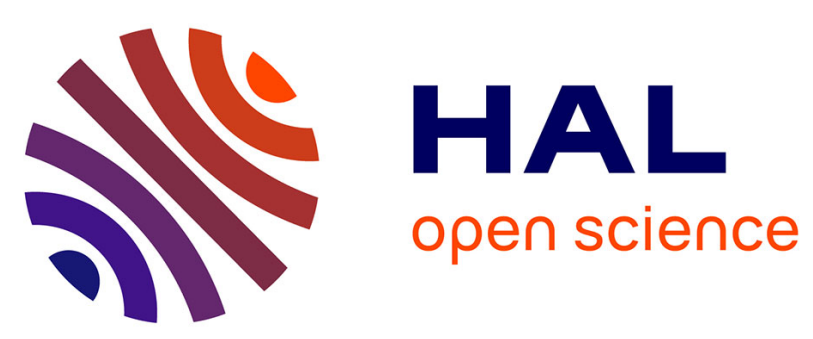

\title{
Widely Linear SC-FDE Systems for Underwater Acoustic Communication: Experimental Results
}

Bruno S. Chang, François-Xavier Socheleau, Christophe Laot

\section{To cite this version:}

Bruno S. Chang, François-Xavier Socheleau, Christophe Laot. Widely Linear SC-FDE Systems for Underwater Acoustic Communication: Experimental Results. OCEANS 2016 - Monterey: MTS/IEEE international conference, Sep 2016, Monterey, United States. pp.1 - 4, 10.1109/OCEANS.2016.7761177 . hal-01424516

\section{HAL Id: hal-01424516 \\ https://hal.science/hal-01424516}

Submitted on 2 Jan 2017

HAL is a multi-disciplinary open access archive for the deposit and dissemination of scientific research documents, whether they are published or not. The documents may come from teaching and research institutions in France or abroad, or from public or private research centers.
L'archive ouverte pluridisciplinaire HAL, est destinée au dépôt et à la diffusion de documents scientifiques de niveau recherche, publiés ou non, émanant des établissements d'enseignement et de recherche français ou étrangers, des laboratoires publics ou privés. 


\title{
Widely Linear SC-FDE Systems for Underwater Acoustic Communication: Experimental Results
}

\author{
Bruno S. Chang ${ }^{1}$, François-Xavier Socheleau ${ }^{2}$ and Christophe Laot $^{2}$ \\ ${ }^{1}$ Electronics Department, Federal University of Technology - Paraná, Curitiba, Brazil \\ Email: bschang@utfpr.edu.br \\ ${ }^{2}$ Institut Mines-Telecom; TELECOM Bretagne, UMR CNRS 6285 Lab-STICC \\ Email: \{fx.socheleau,christophe.laot\}@ telecom-bretagne.eu
}

\begin{abstract}
This work presents experimental results for single carrier underwater communication systems using widely linear frequency domain equalization. The underwater channel models were obtained from a stochastic replay-based channel simulator, which was fed with data from channel measurements conducted in the harbor of Brest (France). It was seen that when symbols from improper constellations are considered it is possible to obtain a performance gain in this scenario for different conditions if widely linear equalization is used.
\end{abstract}

\section{INTRODUCTION}

The underwater environment is one of the toughest ones for data communication. The electromagnetic waves typically used in wireless communications have poor underwater propagation, making acoustic waves the best choice to transmit data in this environment [1]. Also, in this case the bandwidth is a function of distance and frequency, while to further complicate the task of the receiver, the channel is highly selective in frequency and the noise power spectral density is non-white [2]. Thus, efficient equalization performs a vital role in ensuring that higher data rate transmissions in this environment become possible.

Appropriate time-domain equalizers for this environment are long and complex; thus, frequency domain equalization (FDE) is desirable to reduce the computational complexity of this process. The most popular systems where this type of equalization is employed are multicarrier ones. Single carrier systems using frequency-domain equalization (SC-FDE) maintain the convenient low complexity equalization in the frequency domain of multicarrier systems while avoiding their characteristic high peak-to-average power ratio (PAPR) [3], which can be a strain on the transducers used to transmit acoustic waves [4]. SC-FDE systems operating in the underwater environment have been presented in [5], [6].

These systems transmit symbols from a complex quadrature amplitude modulation (QAM) constellation. QAM symbols can be described as proper, that is, their second-order statistics are completely described by the autocorrelation. The PAPR of the transmitted symbols can be further reduced by the usage of symbols from real or offset constellations instead of QAM ones, since their envelope is constant or quasi-constant [7]. These constellations are known as improper. Widely linear (WL) processing takes advantage of the non-vanishing pseudoautocorrelation of these improper sequences to improve the symbols' estimation performance [8], [9]. In order to do that, the received signal must be processed together with its conjugate version. WL processing has been used in SC-FDE systems in [10], [11], [12]. It was seen that widely linear equalizers have better error performance than linear ones, due to their full usage of the available second order statistics, i.e., the autocorrelation together with the pseudoautocorrelation.

This work further develops [13], in which a preliminary analysis of the usage of widely linear equalizers for SCFDE systems in the underwater environment was presented. Differently from [13], in this work underwater channel models were obtained from a stochastic replay-based channel simulator, which was fed with data from channel measurements conducted in the harbor of Brest (France). Replay-based channel simulators are able to generate new random time-varying impulse responses with statistical properties (almost) identical to the original measurement, providing a high degree of realism. It was seen that in this environment SC-FDE systems using WL equalization have better performance than when using linear equalizers, because they are able to exploit the pseudoautocorrelation contained in the transmitted improper sequences.

The remainder of the paper is organized as follows: Section II presents and the system model considered in this paper. In Section III the underwater channel models used in this work are introduced, together with an analysis of the results. Concluding remarks are presented in Section IV.

Notation: vectors are represented by bold lowercase letters, while bold capital letters denote matrices. Time domain elements have a tilde appended to them. The superscripts ()*, ()$^{T}$ and ()$^{H}$ denote, respectively, conjugate, transpose and Hermitian operations. The $i$-th element of the vector $\mathbf{v}$ is given by $v_{i}$ and the $(i, j)$-th element of the matrix $\mathbf{M}$ is given by $M_{i, j}$. The identity matrix of size $N \times N$ is denoted by $\mathbf{I}_{N}$. Finally, the mathematical expectation is $E\{$.$\} .$

\section{SySTEM MODEL}

The system model considered in this paper is presented in Figure 1. Consider a signal block $\tilde{\mathbf{s}}$ of size $N$, whose symbols come from an improper constellation (such as binary phase shift keying (BPSK) or offset quadrature amplitude modulation (OQAM)). Before transmission, a cyclic prefix of length $L_{C P}$ 


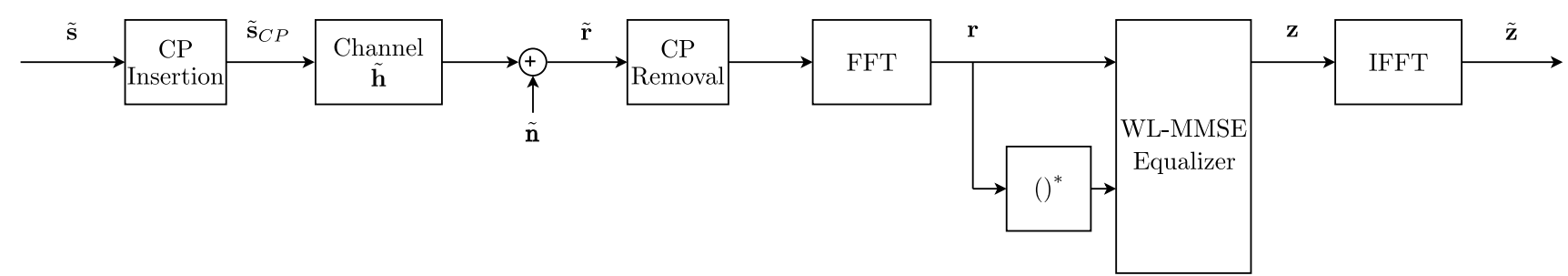

Fig. 1: The system model for a SC-FDE system using a WL-MMSE equalizer.

is appended to the block of symbols, forming $\tilde{\mathbf{s}}_{C P}$ with length $N+L_{C P}$. Before transmission, $\tilde{\mathbf{s}}_{C P}$ will be oversampled.

This symbol block is transmitted through a doubly selective channel model, modeled as a random linear time-varying system that maps an input signal $s(t)$ onto the output $r(t)$ according to the following $\mathrm{I} / \mathrm{O}$ relationship:

$$
\tilde{r}(t)=\int_{\tau} \tilde{h}(\tau, t) \tilde{s}(t-\tau) d \tau+\tilde{n}(t)
$$

where $\tilde{h}(\tau, t)$ is the channel impulse response between the transmitter and the hydrophone and $\tilde{n}(t)$ denotes the ambient noise. We recall that noise in the underwater environment is considered to come mainly from four sources: turbulence, shipping, waves and thermal noise [2].

In the receiver, the signal block is first downsampled; after, it is stripped of its cyclic prefix and converted to the frequency domain. Thus, the vector $\mathbf{r}$ at the input of the equalizer with size $N$ can be expressed as

$$
\mathbf{r}=\mathbf{H} \mathbf{s}+\mathbf{n},
$$

where $\mathbf{H}$ is a $N \times N$ diagonal matrix with its $(k, k)$-th entry $H_{k, k}$ corresponding to the $k$-th coefficient of the $N$-sized DFT of the time domain channel impulse response $\tilde{\mathbf{h}}$, $\mathbf{s}$ is the frequency domain version of $\tilde{\mathbf{s}}$ and the $N$-sized vector $\mathbf{n}$ contains the noise, with variance $\sigma_{n}^{2}$.

Equalization is performed in the frequency domain by filters based on the minimum mean square error (MMSE) criterion. However, since the equalizer is dealing with a signal from an improper constellation (which has non-zero pseudoautocorrelation), it can employ widely linear processing to use all the second-order statistics made available by the received signal and improve its performance. In order to do that, the original version of the received signal in the frequency domain $\mathbf{r}$ together with its conjugate version $\mathbf{r}^{*}$ is processed by a widely linear equalizer $\mathbf{R}_{W L}$, expressed as [11]

$\mathbf{R}_{W L}=\left[\begin{array}{cc}\mathbf{H H}^{H}+\sigma_{n}^{2} \mathbf{I}_{N} & \mathbf{H} \mathbf{U} \mathbf{H}^{T} \\ \mathbf{H}^{*} \mathbf{U H}^{H} & \mathbf{H}^{*} \mathbf{H}^{T}+\sigma_{n}^{2} \mathbf{I}_{N}\end{array}\right]^{-1}\left[\begin{array}{c}\mathbf{H} \\ \mathbf{H}^{*} \mathbf{U}\end{array}\right]$

where $\mathbf{U}$ is given by

$$
\mathbf{U}=\left[\begin{array}{ccccc}
1 & 0 & 0 & \ldots & 0 \\
0 & 0 & 0 & \ldots & 1 \\
\vdots & \vdots & \vdots & \vdots & \vdots \\
0 & 0 & 1 & \ldots & 0 \\
0 & 1 & 0 & \ldots & 0
\end{array}\right]
$$

$\mathbf{R}_{W L}$ can be separated as $\mathbf{R}_{1}$ (which processes the received signal in the frequency domain) and $\mathbf{R}_{2}$ (which processes its conjugate version), which are respectively given by

$$
\mathbf{R}_{1}=\left(\mathbf{H}_{\bmod }+\sigma_{n}^{2} \mathbf{I}_{N}\right)^{-1} \mathbf{H}
$$

and

$$
\mathbf{R}_{2}=\left(\mathbf{H}_{m o d}+\sigma_{n}^{2} \mathbf{I}_{N}\right)^{-1} \mathbf{H}^{*} \mathbf{U}
$$

with

$$
\mathbf{H}_{\text {mod }}=\mathbf{H H}^{H}+\mathbf{U H H}^{H} \mathbf{U} .
$$

Thus, the equalized signal $\mathbf{z}$ can be expressed as

$$
\begin{aligned}
\mathbf{z} & =\mathbf{R}_{1}^{H} \mathbf{r}+\mathbf{R}_{2}^{H} \mathbf{r}^{*} \\
& =\mathbf{R}_{W L}^{H} \mathbf{t},
\end{aligned}
$$

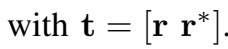

We recall that if this equalizer is dealing with a proper constellation the pseudoautocorrelation is zero and the WL equalizer is reduced to the linear one, expressed by

$$
\mathbf{R}_{L}=\left(\mathbf{H} \mathbf{H}^{H}+\sigma_{n}^{2} \mathbf{I}_{N}\right)^{-1} \mathbf{H} .
$$

Then, the equalized signal $\mathbf{z}$ in the frequency domain passes through an IFFT so that symbol decision is done on the time domain symbol $\tilde{\mathbf{z}}$.

\section{RESULTS}

To obtain impulse responses for the channel model used in this paper, measurements were conducted in the harbor of Brest, France in May 2015 to collect real data. The transmissions were realized between two docks over a 800 $\mathrm{m}$ distance, in a $20 \mathrm{~m}$ water depth. At the transmitter side, an omnidirectional transducer ITC-1032 (resonant frequency: 35 $\mathrm{kHz}$ ) immersed at a depth of $2 \mathrm{~m}$ was used. At the receiver side, a broadband omnidirectional BK-8106 hydrophone was vertically deployed at a depth of $3 \mathrm{~m}$. Estimates of the timevarying channel impulse response (TVIR) were obtained by successive matched filtering to a known probe signal transmitted repeatedly. The probe signal used during the experiments was a m-sequence of 255 BPSK chips transmitted at a symbol rate of $5 \mathrm{kbds}$. Such a sequence can capture arrivals delayed up to $51 \mathrm{~ms}$ and channel estimates can be updated up to 19.6 times per second. Measurements were made at a carrier frequency of $35 \mathrm{kHz}$ and transmitted symbols were pulse-shaped by a root raised cosine filter with a roll-off factor of 0.1. Figure 2 shows the channel estimate obtained in this experiment, while 
Figure 3 shows the average power delay profile of this channel model. It is possible to see in these Figures that the main tap of this channel, which corresponds to the direct path, is quite stable and the rms delay spread is approximately $6.5 \mathrm{~ms}$.

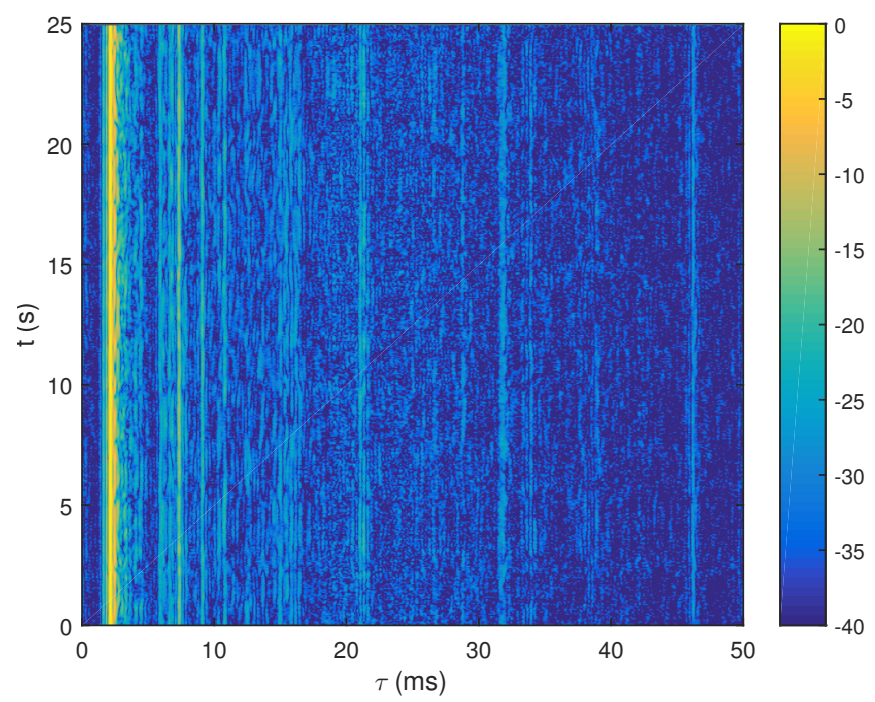

Fig. 2: Channel impulse response for the considered underwater channel.

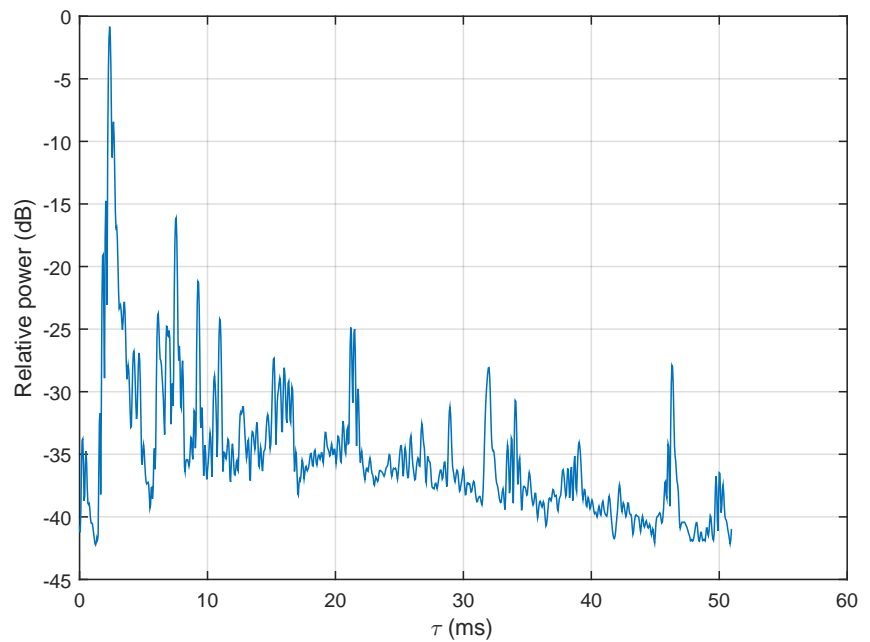

Fig. 3: Average power delay profile for the considered underwater channel.

At the receiver side, the recorded signal is first downconverted into complex-baseband and downsampled. Then, time-varying Doppler shifts are mitigated by an iterative resampling procedure presented in [14].

This measured at sea data is fed to a stochastic replay-based channel simulator [15] to obtain new random TVIRs with statistical properties (almost) identical to the original measurement; these TVIRs are used for the actual simulation. The time-varying Doppler shift of this channel impulse response has been removed in order to mitigate the drift of multipath arrivals that could obscure the analysis of the taps statistics.

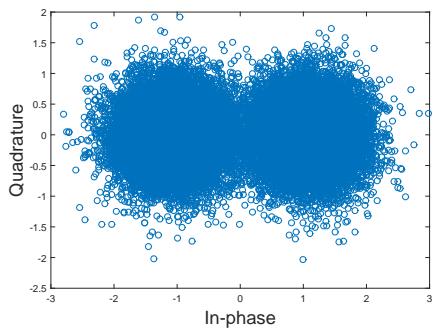

(a) Linear

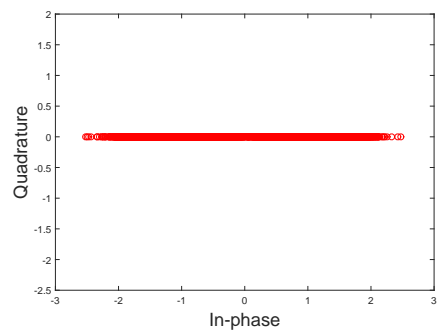

(b) Widely Linear
Fig. 4: Constellation output of the different MMSE equalizers in SC-FDE systems for a SNR of $19 \mathrm{~dB}, N=128$ and $L_{C P}=$ 32.

For the bit error rate (BER) results presented in this section, the carrier frequency $f_{c}$ was $35 \mathrm{kHz}$, the bandwidth $5.5 \mathrm{kHz}$, 100 channel realizations were made and BPSK symbols were transmitted. The experimental conditions at the port allowed us to obtain a SNR of $19 \mathrm{~dB}$ for a transmitted power of $190 \mathrm{~dB}$ re $\mu \mathrm{Pa}$ at $1 \mathrm{~m}$. For this $\mathrm{SNR}$, a block size $N=128$ and a cyclic prefix (CP) $L_{C P}=32$ Figure 4 shows constellations from random symbols at the output of the SC-FDE systems when using a linear equalizer (in Figure 4a) and a widely linear equalizer (Figure 4b). A BER of $2.3 \times 10^{-3}$ was obtained when using the linear equalizer, while when using widely linear equalization the BER value was $3.4 \times 10^{-4}$ for this scenario. This performance gain is due to the fact that the receiver employing widely linear processing takes into account all of the available second order statistics when processing the improper received signal, leading to a better estimate.

An interesting characteristic when using the stochastic replay method to obtain the channel impulse responses is that it is possible to change the SNR values for a given TVIR. With this feature it is possible to test the WL-MMSE equalizer with other SNR values, to see if the performance gain seen above is upheld. Figure 5 presents a comparison of the error performance of these systems for different values of SNR, again with $N=128$ and $L_{C P}=32$. As it is possible to see in this Figure, the WL performance gain is obtained throughout the considered SNR range.

Finally, in Figure 6 the impact of the block size $N$ on the error performance of these systems is seen. For these results, the $\mathrm{CP}$ length was fixed at $L_{C P}=64$ and the values of $N$ considered were 256 and 512. An increase of the block size brings better error performance for both systems, because this larger block size allows the MMSE equalizers in SC-FDE systems to better harness the available diversity [16]. For both block sizes there is a performance advantage for the systems employing widely linear processing.

\section{CONCLUding REMARKS}

In this work, results from SC-FDE systems using widely linear MMSE equalization operating with channel impulse responses coming from a replay-based simulator fed with realistic underwater acoustic channel models were presented. 


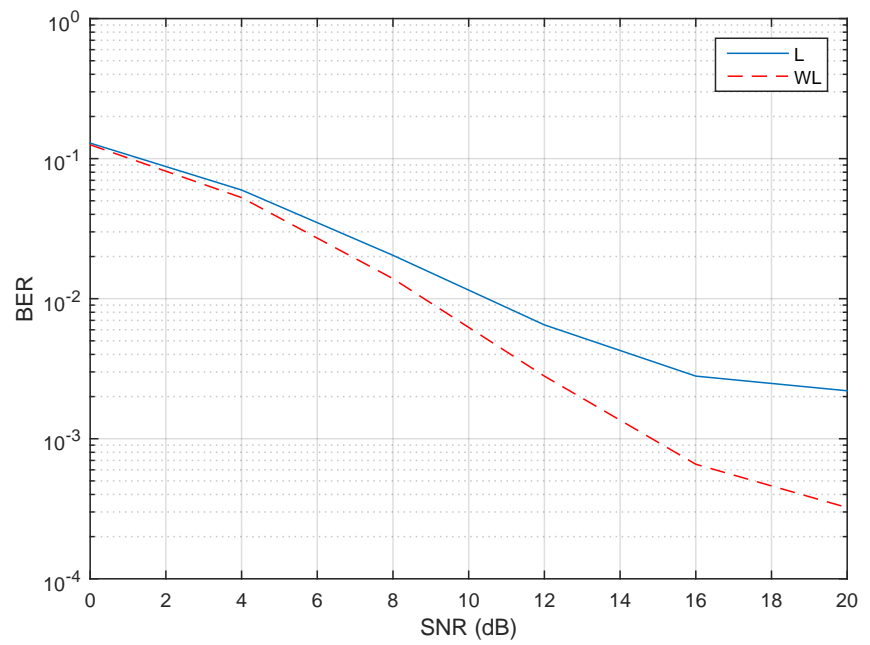

Fig. 5: Error performance comparison for $N=128$ and $L_{C P}=32$.

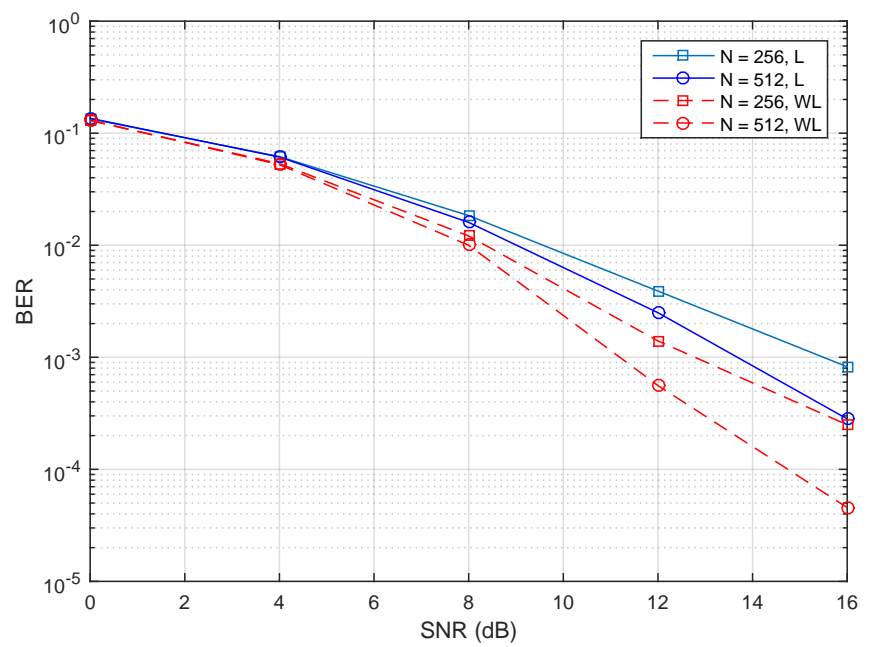

Fig. 6: Error performance comparison for $L_{C P}=64$ and different block sizes.

These realistic CIRs were obtained in Brest harbor, France. It is possible to see that when transmitting improper sequences the usage of widely linear equalization brings a performance gain with respect to systems using linear equalizers, due to the fact that full second-order statistics are taken into account in the equalization process. For a carrier frequency of $35 \mathrm{kHz}$ and a bandwidth of $5.5 \mathrm{kHz}$ the system using WL equalization has a single-channel average BER of $3.4 \times 10^{-4}$ with BPSK modulation for a experimental SNR of $19 \mathrm{~dB}, N=128$ and $L_{C P}=32$; this BER was better than the one obtained when using linear equalization $\left(2.3 \times 10^{-3}\right)$. For other values of SNR and block size there is also a performance advantage when using WL equalization. Future developments of this work could include a performance evaluation using multiple hydrophones in the receiver [17] and/or using iterative widely linear frequency domain equalization [18].

\section{ACKNOWLEDGMENT}

This work has been partially sponsored by CNPq (Brazil).

\section{REFERENCES}

[1] R. Urick, Principles of Underwater Sound, 3rd ed. McGraw Hill, 1996.

[2] M. Stojanovic, "On the relationship between capacity and distance in an underwater acoustic communication channel," ACM SIGMOBILE Mobile Computing and Communications Review, vol. 11, no. 4, pp. 3443, 2007.

[3] D. Falconer, S. Ariyavisitakul, A. Benyamin-Seeyar, and B. Eidson, "Frequency domain equalization for single-carrier broadband wireless systems," IEEE Communications Magazine, vol. 40, no. 4, pp. 58-66, Apr. 2002.

[4] C. He, J. Huang, Q. Zhang, and X. Shen, "Single carrier frequency domain equalizer for underwater wireless communication," in Cоттиnications and Mobile Computing, 2009. CMC'09. WRI International Conference on, vol. 1. IEEE, 2009, pp. 186-190.

[5] A. Youcef, C. Laot, and K. A. Cavalec, "Single carrier frequency division multiple access for underwater acoustic communications," in UA 2013. 1 st international conference on Underwater Acoustics, 2013.

[6] M. Xia, D. Rouseff, J. Ritcey, X. Zou, C. Polprasert, and W. Xu, "Underwater acoustic communication in a highly refractive environment using sc-fde," Oceanic Engineering, IEEE Journal of, vol. 39, no. 3, pp. 491-499, July 2014.

[7] M. Luzio, R. Dinis, and P. Montezuma, "On the Use of Multiple Grossly Nonlinear Amplifiers for an Efficient Amplification of OQAM Signals with FDE Receivers," in Vehicular Technology Conference (VTC Fall), 2011 IEEE, Sep. 2011.

[8] P. Schreier and L. Scharf, "Second-order analysis of improper complex random vectors and processes," IEEE Transactions on Signal Processing, vol. 51, no. 3, pp. 714-725, Mar. 2003.

[9] P. Chevalier and F. Pipon, "New Insights Into Optimal Widely Linear Array Receivers for the Demodulation of BPSK, MSK, and GMSK Signals Corrupted by Noncircular Interferences - Application to SAIC," IEEE Transactions on Signal Processing, vol. 54, no. 3, pp. 870-883, Mar. 2006.

[10] Z. Lin, P. Xiao, B. Vucetic, and M. Sellathurai, "Analysis of receiver algorithms for LTE SC-FDMA based uplink MIMO systems," IEEE Transactions on Wireless Communications, vol. 9, no. 1, pp. 60-65, Jan. 2010.

[11] B. Chang, C. da Rocha, D. Ruyet, and D. Roviras, "Widely linear mmse precoding and equalization techniques for sc-fde systems," EURASIP Journal on Advances in Signal Processing, vol. 2014, no. 1, p. 124, 2014.

[12] M. Kim, J. H. Cho, and J. S. Lehnert, "Asymptotically optimal lowcomplexity sc-fde with noise prediction in data-like improper-complex interference," IEEE Transactions on Wireless Communications, vol. 15, no. 3, pp. 2090-2103, 2016.

[13] B. S. Chang, R. D. Souza, and M. E. Pellenz, "On the performance of widely linear sc-fde systems for underwater acoustic communication," in OCEANS 2015-Genova. IEEE, 2015, pp. 1-4.

[14] P. A. Van Walree, T. Jenserud, and M. Smedsrud, "A discrete-time channel simulator driven by measured scattering functions," IEEE journal on selected areas in communications, vol. 26, no. 9, pp. 1628-1637, 2008.

[15] F.-X. Socheleau, C. Laot, and J.-M. Passerieux, "Stochastic replay of non-wssus underwater acoustic communication channels recorded at sea," Signal Processing, IEEE Transactions on, vol. 59, no. 10, pp. 4838-4849, 2011.

[16] A. Tajer and A. Nosratinia, "Diversity Order in ISI Channels with Single-Carrier Frequency-Domain Equalizers," IEEE Transactions on Wireless Communications, vol. 9, no. 3, pp. 1022-1032, 2010.

[17] F.-X. Socheleau, A. Pottier, and C. Laot, "Stochastic replay of simo underwater acoustic communication channels," in OCEANS 2015MTS/IEEE Washington. IEEE, 2015, pp. 1-6.

[18] B. S. Chang, C. A. da Rocha, D. Le Ruyet, and D. Roviras, "Widely linear iterative equalizers for sc-fde systems," in Wireless Communication Systems (ISWCS 2013), Proceedings of the Tenth International Symposium on. VDE, 2013, pp. 1-5. 